\title{
Adaptive Localisation for Unmanned Surface Vehicles Using IMU-Interacting Multiple Model
}

\author{
Bryan Adam Gunawan \\ Dept. Mechanical Engineering \\ University College London \\ London, UK \\ bryan.gunawan.17@ucl.ac.uk
}

\author{
Yuanchang Liu \\ Dept. Mechanical Engineering \\ University College London \\ London, UK \\ yuanchang.liu@ucl.ac.uk
}

\author{
Xudong Li \\ Key Laboratory for Micro/Nano \\ Technology and System of Liaoning \\ Province \\ Dalian University of Technology \\ Dalian, China \\ lixudong2015@mail.dlut.edu.cn
}

\begin{abstract}
Unscented Kalman Filter (UKF) remains to be a prevalent multi-sensor fusion method in many practices, including navigational tracking for Unmanned Surface Vehicles (USVs). This paper suggests that results from UKF fusion is unsatisfactory for USVs' relatively smooth path due to UKF's lack of versatility. Hence, it is proposed here that by replacing the UKF with Interacting Multiple Model (IMM), estimation results will better represent USV's movement. Furthermore, this paper proposes slight modification to the IMM in order to heighten the algorithm's confidence in switching modes. By exploiting angular velocity information from Inertial Measurement Unit (IMU), an independent mode probability can be obtained which is then injected into the IMM. Computer simulations based on maritime operations were done to show that the proposed IMU-based IMM is able to react faster to mode changes, giving more reliable outcomes.
\end{abstract}

Keywords-Interacting Multiple Model (IMM), adaptive estimation, USV navigation, GPS/IMU

\section{INTRODUCTION}

\section{A. Background \& Problem Statement}

With the growing interest in Unmanned Surface Vehicles (USVs), more effort in research are being placed into the field. Similar to other autonomous vehicles, self-navigation is fundamentally based on localisation, path-planning and control. As localisation is one integral part of autonomy, it remains to be an aspect to be optimally addressed.

Global Positioning System (GPS), Inertial Measurement Unit (IMU) and electronic compass are low-cost and highly effective combination of sensors widely found installed in USVs $[1,2,3,4]$. The main issue of localisation stems from measurement uncertainties that each sensor carries. Added with environmental disturbances, raw data cannot be relied fully. Readings from all sensors need to be fused and filtered to produce useful information for USVs navigation. A robust algorithm that is able to take in raw data and produce good state estimates is therefore needed.

Making physical connection between the sensors to its processor is detailed in [4] and [5]. While both papers prescribe their experimental apparatus containing GPS/IMU \& compass sensor fusion along with its processor, the latter paper applies it more specifically on USVs. This paper follows the laid-out assembly and aims to bridge in the knowledge gap by proposing an improved algorithm for USVs' localisation.

\section{B. Literature Review}

For the problem of data fusion, Kalman Filter (KF) is undoubtedly an acknowledged method for linear problems. State estimates are calculated recursively by passing a priori state through an appropriate dynamic model, then updating it with new measurement state. Two states are combined in a maximum likelihood sense considering both its state and defined noise covariances [6]. KF has been well accepted for integrated GPS/IMU fusion use [7]. However, application of linear problems is limited in practical scenarios and the nature of fusing multiple sensors itself may introduce nonlinearity. So, for problems indescribable with linear systems, nonlinear variants of KF are developed.

Out of many nonlinear filters, Unscented Kalman Filter (UKF) is a highly respected algorithm [8]. Compared to its alternatives, UKF offers a more robust approximation to any nonlinear cases and simpler formulation. This is achieved by propagating a set of Sigma points through the actual nonlinear function. Newly transformed Sigma points are then recalculated to form the new covariance. Taking advantage of $\mathrm{UKF}$, significant attention has been placed in applying the algorithm for USVs' navigational purposes with GPS/IMU. $[4,6,9,10]$. Other equally notable ways like Particle filter is ruled out of interest in limiting computational burden.

Following these progresses, adaptive features are being introduced to enhance functionality. There are a few types of adaptive techniques, but of all, covariance matching is the most efficient for real-time computation [11, 12, 13]. Covariance matching is based on online noise covariances revision, mitigating imprecise priori knowledge that may lead into deteriorating results [14]. Reference [15] propound a covariance matching UKF by adjusting both covariances to actual value. Zheng et al. [16] takes a more particular approach by devising a statistical switch that detects necessity in making changes. In [17], the authors demonstrate effectiveness of using fuzzy logic to match measurement covariance in USV tracking. There are countless other methods; e.g. see [3, 11, 18, 19, 20, 21, 22]. Such benefits from solely adapting covariances, however, are observable only when covariances differ from initially defined. Furthermore, these features typically entail additional parameters, undermining the term 'adaptive' which ideally should adjust automatically to various conditions with least manual interventions or manual definitions. Most importantly, the emphasis of the problem statement here is that sticking to a single model algorithm is a major downside in a chaotic environment.

Efforts to use multiple-model (MM) approach in USVs application has interestingly not been deeply explored. Dynamic behaviour in USVs operation can be opportunely split into two modes: straights and turns. To only use model beneficial for changes in direction will ensue responsive but noisy results. On the other hand, using model favourable for straights brings smoothness but lags in capturing turns. Most single model methods depend on acceleration-based models, understandably prioritising responsiveness over smoothness. Reference [2] takes in Multiple Model Adaptive Estimation into its USVs navigational system and has shown its potency. More recent works have presented that Interacting Multiple 
Model (IMM) [23] outperforms other MM methods. IMM's ability to efficiently switch between different models appeals to many tracking scenarios. Work in [24] has proven effective usability of IMM in maritime tracking environment, but not with GPS/IMU combination. Variable Structure Multiple Model (VSMM) is a more recent advancement to the IMM [25]. Large number of models in IMM impose great computational demand. VSMM overcomes this by having a set of defined models to select from. The fewer chosen models are later used in the main MM algorithm, removing unnecessary 'competitions' from statistically irrelevant models. Although VSMM is computationally more beneficial, current developments indicate no significant superiority in estimation accuracy compared to IMM. Advantages of VSMM are apparent when numerous possible models are needed. In this case, with only two models, the more complex VSMM has nothing to offer over IMM. [26, 27, 28, 29, 30]

Past works on adaptive GPS/IMU fusion uses accuracy as its primary scoring system. While smoothness, an essential attribute to have for USVs' localisation, remains underappreciated. Achieving accurate and smooth results necessitate the use of multiple models than UKF alone. This paper demonstrates that IMM can lead to a more realistic estimation than the conventional UKF. Moreover, novel adjunctive adjustment based on the incorporation of independent statistics derived from IMU into the mode probability update step in IMM is proposed to enhance its adaptability. Since the amendments put forth is minor with nearly no additional parameters than the original, IMM's integrity and efficiency are still maintained.

The structure of this paper is distributed as follows. Section II briefly details model associated in USVs and Section III describes IMM framework in which proposed algorithm is built on. Followed by explanations on the added mechanism in Section IV. Evidence to support the hypothesis are presented through simulation results and their respective assessments in Section V. The paper is then concluded in Section VI with remarks regarding existing limitations and potential future work.

\section{UNMANNED SURFACE VEHICLES NAVIGATION}

The purpose of localisation in USVs is to provide valuable information of current state to other important aspects in the system. GPS, IMU and electronic compass are sensors that serves into mentioned state data, namely position $(p)$, velocity $(v)$ and heading $(\theta)$. Defining state as $\boldsymbol{x}$, it can therefore be written in a vector form as:

$$
\boldsymbol{x}=\left[\begin{array}{lllll}
p_{x} & p_{y} & v_{x} & v_{y} & \theta
\end{array}\right]^{\mathrm{T}}
$$

Disturbances from installed sensors can be assumed to be constituted into three parts: true value, white noise and bias. Overall acceleration and angular velocity value $\left(a_{o}\right.$ and $\left.\omega_{o}\right)$ are modelled from its true value $\left(a_{i}\right.$ and $\left.\omega_{i}\right)$ added by random gaussian noise $\left(w_{a}\right.$ and $\left.w_{g}\right)$ and bias $\left(b_{a}\right.$ and $\left.b_{g}\right)$. Statistical uncertainty for the random gaussian noise is provided by manufacturers.

Generally, GPS and electronic compass are not associated with bias. Henceforth, their readings are represented by true position and heading value $\left(p_{i}\right.$ and $\left.\theta_{i}\right)$ and random zero-mean gaussian noise $\left(v_{p}\right.$ and $\left.v_{\theta}\right)$.

$$
\begin{gathered}
a_{o}=a_{i}+b_{a}+w_{a} \\
\omega_{o}=\omega_{i}+b_{g}+w_{g} \\
p_{o}=p_{i}+v_{p} \\
\theta_{o}=\theta_{i}+v_{\theta}
\end{gathered}
$$

where $w_{a}, w_{g}, v_{p}$ and $v_{\theta}$ are gaussian noises representing their own uncertainty $\sigma^{2}$ of distribution $N\left(0, \sigma^{2}\right)$. The algorithm's main purpose is hence to provide information for state $\boldsymbol{x}$ despite measurement uncertainties by filtering out associated noises.

\section{INTERACTING MULTIPLE MODEL (IMM)}

IMM consists of multiple recursive algorithms running in parallel that interacts with each other using Markovian coefficient. At every step, probability for every possible mode transition is calculated in a Markovian manner to manipulate a bank of multiple models such that the more relevant model for current condition is endorsed. Two modes of USVs, straights and turns, are represented by Constant Velocity Model (CVM) and Coordinated Turn Model (CTM), respectively.

CVM best characterise the dynamics of USVs when it is undergoing constant velocity and constant heading. The discretised equation is defined as follows:

$$
x_{k}=F_{C V M} x_{k-1}+w_{k-1}
$$

where $\boldsymbol{w}_{\boldsymbol{k}-\mathbf{1}}$ is the process noise covariance assumed to be a random gaussian noise with $p(\boldsymbol{w}) \sim N(0, \boldsymbol{Q})$ with $\boldsymbol{Q}$ being the process noise covariance and $\boldsymbol{F}_{\boldsymbol{C V} \boldsymbol{M}}$ is the CVM prediction model.

$$
\boldsymbol{F}_{C V M}=\left(\begin{array}{ccccc}
1 & 0 & T & 0 & 0 \\
0 & 1 & 0 & T & 0 \\
0 & 0 & 1 & 0 & 0 \\
0 & 0 & 0 & 1 & 0 \\
0 & 0 & 0 & 0 & 1
\end{array}\right)
$$

USVs are rather restricted in its rotational movements. During turns, CTM best portrays this limitation as it is derived from a constant velocity rotation along a circular path; a more realistic dynamics for surface vehicles. Comprehensive derivation of the model is expounded in [28] and the set of equations for CTM is shown below:

$$
x_{k}=F_{C T M}(\omega) x_{k-1}+w_{k-1}
$$

with

$$
\left(\begin{array}{ccccc}
1 & 0 & \frac{\sin (\omega T)}{\omega} & -\frac{1-\cos (\omega T)}{\omega} & \boldsymbol{F}_{C T M}(\omega)= \\
0 & 1 & \frac{1-\cos (\omega T)}{\omega} & \frac{\sin (\omega T)}{\omega} & 0 \\
0 & 0 & \cos (\omega T) & -\sin (\omega T) & 0 \\
0 & 0 & \sin (\omega T) & \cos (\omega T) & 0 \\
0 & 0 & 0 & 0 & 1+\frac{\omega T}{x_{\theta, k-1}}
\end{array}\right)
$$


Selected models then need to be mathematically connected together by the IMM. Below elaborates the mechanism in which IMM operates. It starts by normalising new mode probability for current step:

$$
\begin{gathered}
\boldsymbol{\mu}_{k \mid k-1}^{i}=\sum_{j} \boldsymbol{\pi}_{j i} \boldsymbol{\mu}_{k-1}^{j} \\
\boldsymbol{\mu}_{k-1}^{j \mid i}=\boldsymbol{\pi}_{j i} \boldsymbol{\mu}_{k-1}^{j} / \boldsymbol{\mu}_{k \mid k-1}^{i}
\end{gathered}
$$

where $\boldsymbol{\pi}_{j i}$ is the probability matrix of switching from mode $j$ to mode $i$ and $\boldsymbol{\mu}_{k-1}^{j}$ is the probability of mode $j$ at time step $k-1$. Mixed input state $\overline{\boldsymbol{x}}_{k-1 \mid k-1}^{i}$ and its covariance $\overline{\boldsymbol{P}}_{k-1 \mid k-1}^{i}$ for each model $i$ are then calculated from previous a posteriori and the normalised mixing probability $\boldsymbol{\mu}_{k-1}^{j \mid i}$.

$$
\begin{gathered}
\overline{\boldsymbol{x}}_{k-1 \mid k-1}^{i}=\sum_{j} \widehat{\boldsymbol{x}}_{k-1 \mid k-1}^{j} \boldsymbol{\mu}_{k-1}^{j \mid i} \\
\overline{\boldsymbol{P}}_{k-1 \mid k-1}^{i}=\sum_{j}\left[\boldsymbol{P}_{k-1 \mid k-1}^{j}+\left(\overline{\boldsymbol{x}}_{k-1 \mid k-1}^{i}-\right.\right. \\
\left.\left.\widehat{\boldsymbol{x}}_{k-1 \mid k-1}^{j}\right)\left(\overline{\boldsymbol{x}}_{k-1 \mid k-1}^{i}-\widehat{\boldsymbol{x}}_{k-1 \mid k-1}^{j}\right)^{T}\right] \boldsymbol{\mu}_{k-1}^{j \mid i}
\end{gathered}
$$

Calculated input states and sensor input $\boldsymbol{z}_{k}$ are then passed through the prediction-update step in each filter model $i$. Posteriori likelihood $\boldsymbol{L}_{k}^{i}$ is also taken for proceeding stages.

$$
\left[\widehat{\boldsymbol{x}}_{k \mid k}^{i}, \boldsymbol{P}_{\mathrm{k} \mid \mathrm{k}}^{\mathrm{i}}\right]=K F\left(\overline{\boldsymbol{x}}_{k-1 \mid k-1}^{i}, \overline{\boldsymbol{P}}_{k-1 \mid k-1}^{i}, \boldsymbol{z}_{k}, f^{i}(\cdot), \boldsymbol{H}^{i}, \boldsymbol{Q}^{i}, \boldsymbol{R}^{i}\right)
$$

$$
\boldsymbol{L}_{k}^{i}=\mathcal{N}\left(\boldsymbol{y}_{k}^{i}: 0, \boldsymbol{S}_{k}^{i}\right)
$$

where $K F(\cdot)$ denotes the whole $\mathrm{KF}$ prediction-update process. $\boldsymbol{y}_{k}^{i}$ is the residual and $\boldsymbol{S}_{k}^{i}$ is the system covariance of model $i$. Likelihood function $\mathcal{N}(\cdot)$ is defined as follows:

$$
\mathcal{N}(\cdot)=\frac{1}{\sqrt{2 \pi S}} \exp \left(-\frac{y S^{-1} y^{\mathrm{T}}}{2}\right)
$$

IMM determines current model probability for time step $k$ with the attained a posteriori likelihood as follows:

$$
\boldsymbol{\mu}_{k}^{i}=\frac{\boldsymbol{\mu}_{k \mid k-1}^{i} \boldsymbol{L}_{k}^{i}}{\sum_{j} \boldsymbol{\mu}_{k \mid k-1}^{j} \boldsymbol{L}_{k}^{j}}
$$

Finally, updated model probability and state posteriori from each model is fused to compute final IMM estimate.

$$
\begin{gathered}
\widehat{\boldsymbol{x}}_{k \mid k}=\sum_{i} \widehat{\boldsymbol{x}}_{k \mid k}^{i} \boldsymbol{\mu}_{k}^{i} \\
\boldsymbol{P}_{k \mid k}=\sum_{i}\left[\boldsymbol{P}_{k \mid k}^{i}+\left(\widehat{\boldsymbol{x}}_{k \mid k}-\widehat{\boldsymbol{x}}_{k \mid k}^{\mathrm{j}}\right)\left(\widehat{\boldsymbol{x}}_{k \mid k}-\widehat{\boldsymbol{x}}_{k \mid k}^{\mathrm{j}}\right)^{\mathrm{T}}\right] \boldsymbol{\mu}_{k}^{i}
\end{gathered}
$$

\section{IMU-BASED IMM}

Reconfigurations of IMM that makes IMU-IMM are done to improve sensitivity to mode changes. Modes of USVs are correlated to angular velocity. Angular velocity is expected to be zero when there is no change in heading during straights; and non-zero during turns. Compliant with the structure of IMM, the angular velocity has to be registered in a probabilistic sense. Likelihood function $\mathcal{N}\left(\omega_{k}: 0, a \sigma_{\omega}^{2}\right)$ satisfies this by placing the measurement in a normal distribution with variance $a \sigma_{\omega}^{2}$; where $a$ is a constant that regulates its sensitivity and $\sigma_{\omega}$ is the specified angular velocity variance from manufacturer's data.

$$
\begin{gathered}
\boldsymbol{L}_{k}^{i}=\mathcal{N}\left(\boldsymbol{y}_{k}^{i}: 0, \boldsymbol{S}_{k}^{i}\right) \\
\boldsymbol{\mu}_{k}^{i}=\frac{\boldsymbol{\mu}_{k \mid k-1}^{i} \boldsymbol{L}_{k}^{i}}{\sum_{j} \boldsymbol{\mu}_{k \mid k-1}^{j} \boldsymbol{L}_{k}^{j}} \\
\mathcal{L}_{k}^{1}=\frac{\mathcal{N}\left(\omega_{k}: 0, a \sigma_{\omega}^{2}\right)}{\mathcal{N}\left(0: 0, a \sigma_{\omega}^{2}\right)} \\
\mathcal{L}_{k}^{2}=1-\mathcal{L}_{k}^{1}
\end{gathered}
$$

Equation (20) and (21) above are identical to (15) and (17) included to indicate where the equations would be added into. According to the likelihood function defined by $a \sigma_{\omega}$, variable $\mathcal{L}_{k}^{1}$ which corresponds to CVM would have a higher normalised value than $\mathcal{L}_{k}^{2}$ given that IMU input $\omega_{k}$ is close to zero - going straight. Otherwise, $\mathcal{L}_{k}^{2}$ will be more dominant than $\mathcal{L}_{k}^{1}$. These normalised values are stored in the matrix $\mathcal{L}_{k}^{i}$. Assimilation of mentioned IMU-based mode likelihood with IMM's probabilistic calculation of $\boldsymbol{\mu}_{k}^{i}$ from (17) increases the 'confidence' of the algorithm in choosing most relevant model. Equation below shows the mixing and normalisation of the mode probabilities.

$$
\boldsymbol{\mu}_{k}^{i}=\frac{\boldsymbol{\mu}_{k}^{i} \mathcal{L}_{k}^{i}}{\sum_{j} \boldsymbol{\mu}_{k}^{j} \mathcal{L}_{k}^{j}}, \quad i=1,2
$$

A better poised final model probability for current time step is acquired through this modification. Revised $\boldsymbol{\mu}_{k}^{i}$ is lastly mixed with posteriori produced by multiple models in IMM in (18) and (19) to yield final estimate. Minor restructuring of IMM also minimally affects IMM's attractive computational efficiency, while still refining its confidence in performing mode switches. Fig. 1 lays out the newly proposed adaptation of the IMM, called as the IMU-based IMM here.

\section{Simulations AND DISCUSSIONS}

\section{A. Set $U p$}

Quantitative analysis on stated improvements are supplied by computer simulations. A series of directional commands are instructed into the USV. Ground truth data are generated based on this input. Constraints to USV's rotational behaviour and acceleration is applied into the simulation, implying a more realistic pathing. As the iteration progresses, USV measurement readings are modelled by adding appropriate gaussian noise to the existing true state. The simulated noisy sensor data are then passed into various filters, enabling assessments of filtering quality. Fidelity of simulation used to depict pragmatic operations is limited by a few assumptions:

- Manufacturer's technical data is taken at value.

- Environmental disturbances are modelled by adding extra noise on top of sensor noise.

- Ground truth behaves in a constant body velocity.

Heading angle is not emphasised in this study due to its remarkably low noise relative to other states as seen from Table I [17]. 


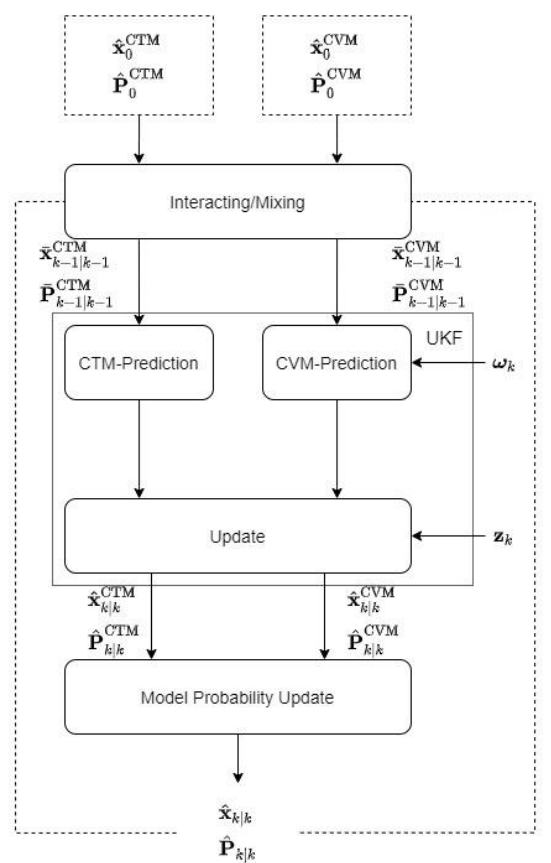

Fig. 1. IMU-IMM Block diagram.

TABLE I. SimUlation SENSOR NOISE Characteristics

\begin{tabular}{cccc}
\hline \multirow{2}{*}{ Sensor } & Measurement & \multicolumn{2}{c}{ Noise } \\
\cline { 3 - 4 } & \multirow{2}{*}{ IMU } & $\begin{array}{c}\text { Standard } \\
\text { Deviation }\end{array}$ & Bias \\
\cline { 2 - 4 } & $\begin{array}{c}\text { Acceleration }\left(a_{i x}\right. \\
\left.\text { and } a_{i y}\right)\end{array}$ & $0.0042 \mathrm{~ms}^{-2}$ & $0.03 \mathrm{~ms}^{-2}$ \\
& $\begin{array}{c}\text { Angular velocity } \\
(\omega)\end{array}$ & $0.036^{\circ} / \mathrm{s}$ & $0.28^{\circ} / \mathrm{s}$ \\
\hline \multirow{2}{*}{ GPS } & $\begin{array}{c}\text { Position }\left(p_{x} \text { and }\right. \\
\left.p_{y}\right)\end{array}$ & $8 \mathrm{~m}$ & \\
\hline Compass & Heading $(\theta)$ & $0.8^{\circ}$ & \\
\hline
\end{tabular}

Accuracy is quantified by calculating the Root Mean Square Error (RMSE), obtaining the absolute distance between filtered results and ground truth. As for smoothness, its judgement is related to changes in error. Independent to accuracy, greater change in error hints lack of smoothness. Root Mean Square Changes in Error (RMSCE) is referred to here to analyse smoothness. Difference to preceding recorded error is taken to give the change in error. Over time, the values are averaged to yield useful numerical descriptions.

$$
\begin{gathered}
\operatorname{RMSE}=\frac{\sum_{i=1}^{n} \sqrt{\left(\boldsymbol{x}_{i}-\widehat{\boldsymbol{x}}_{i \mid i}\right)^{2}}}{n} \\
\operatorname{RMSCE}=\frac{\sum_{i=2}^{n} \sqrt{\left(\left(\boldsymbol{x}_{i}-\widehat{\boldsymbol{x}}_{i \mid i}\right)-\left(\boldsymbol{x}_{i-1}-\widehat{\boldsymbol{x}}_{i-1 \mid i-1}\right)\right)^{2}}}{n-1}
\end{gathered}
$$

where $n$ is the total step taken by the autonomous operation, $\boldsymbol{x}_{i}$ denotes simulated true state during step $i$ and $\widehat{\boldsymbol{x}}_{i \mid i}$ defines final estimated output from the algorithm.

\section{B. Scenario 1}

Scenario 1 begins by testing filter performances in a relatively straight route. Slight curvatures in the path are added to simulate course corrections that may occur in an autonomous operation. Programmed commands involve a small right turn followed by an adjustment to the left.
Fig. 2 and Fig. 3 displays positional estimates of different algorithms given readings from perturbed sensors. Ground truth and GPS readings are shown in Fig. 2 as black line and blue dots, respectively. The same figure overlaps IMU-IMM (orange-line), IMM (green line) and conventional UKF (red line) for comparison. In the enlarged inset, UKF is seen to lie close to true state but struggles in keeping smoothness even in relatively straight path. Abrupt changes in UKF reacts quickly to deviations, but in a notably unrealistic way. In contrast, IMM stays consistently apart but without dramatic changes. IMU-IMM inherits the two benefits, retaining IMM's smoothness while keeping UKF's accuracy.

Fig. 3 shows how IMU-IMM fares in error against other filters; both positional and velocity errors are maintained at low magnitude. Taking average of RMSE over the whole course, IMU-IMM does about $50 \%$ and $70 \%$ better than UKF in position and velocity estimation, respectively. Compared to IMM, IMU-IMM is around $30 \%$ better in position and $15 \%$ better in velocity estimation. About smoothness, IMU-IMM is expectedly superior to UKF by $50 \%$ and $75 \%$ in position and velocity, respectively. Whereas marginal drop in smoothness performance from IMM to IMU-IMM can be observed at approximately $5 \%$ worse for each state of interest. This can be explained by accounting IMU-IMM's higher degree of flexibility in tolerating possible changes. This actually shows that IMU-IMM can reasonably keep IMM's desirable quality, while tremendous RMSCE discrepancy to UKF affirms improved reliability. Scenario 1 clearly reveals IMU-IMM's benefits over conventional UKF.

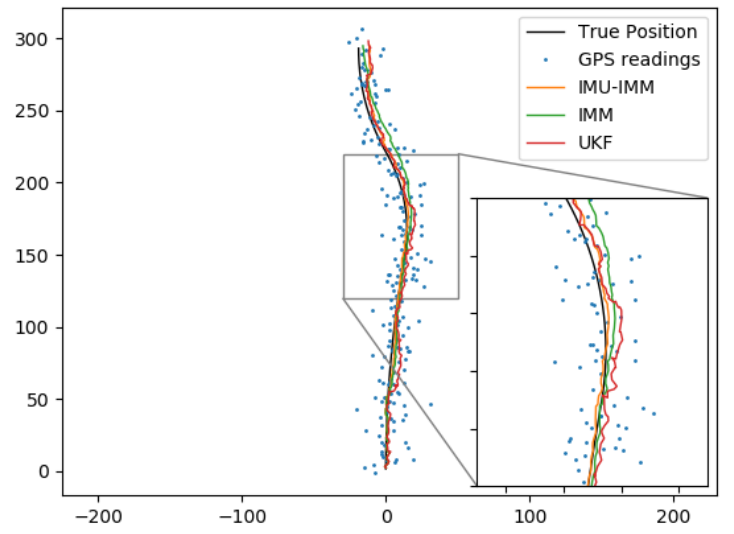

Fig. 2. Scenario 1: Position results for IMU-IMM, IMM and UKF.
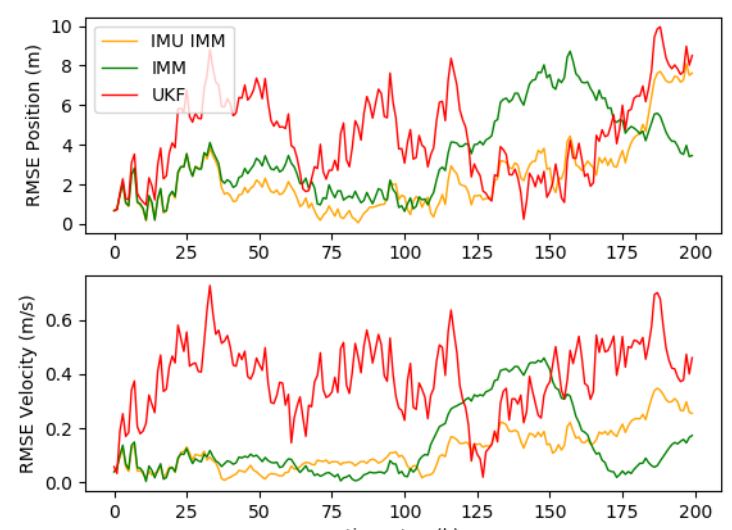

Fig. 3. Scenario 1: Position \& Velocity RMSE for IMU-IMM, IMU and UKF. 


\section{Scenario 2}

The aim of Scenario 2 is to simulate a more practical autonomous drive. Route planned by USVs usually involve numerous adjustments to avoid detected obstacles. Arbitrary turns are introduced in Scenario 2 to imitate this effect. This simulation initialises straight up North from origin, before entering a right turn into a curved forward movement, emulating current effects. The path ends with two consecutive right turns continued by a left to return the USV.

Fig. 4 and 5 adheres to the same format as of figures in Scenario 1. Consistent to previous discussion, red line (UKF) in Fig. 4 sustains satisfactory accuracy throughout, although it is unstable even during. Such accuracy is matched with orange line (IMU-IMM) but with comparable smoothness to green line (IMM). IMM's smooth estimation lags behind in turns before eventually merging back closer to ground truth. The figure exhibits IMU-IMM's aptitude in giving smooth and accurate localisation under unpredictable manoeuvres. This ability is granted through the incorporation of angular velocity input to emend the posteriori mode matrix.

Error plot is now less consistent as the scenario involves irregularities, testing different properties. IMM's incapability to immediately capture mode switches is further hinted by its error peak during turns. Meanwhile, errors in UKF oscillates persistently at about $4 \mathrm{~m}$ and $0.3 \mathrm{~m} / \mathrm{s}$, suggesting consistency without stability. IMU-IMM displays the best trade-off between the two properties.

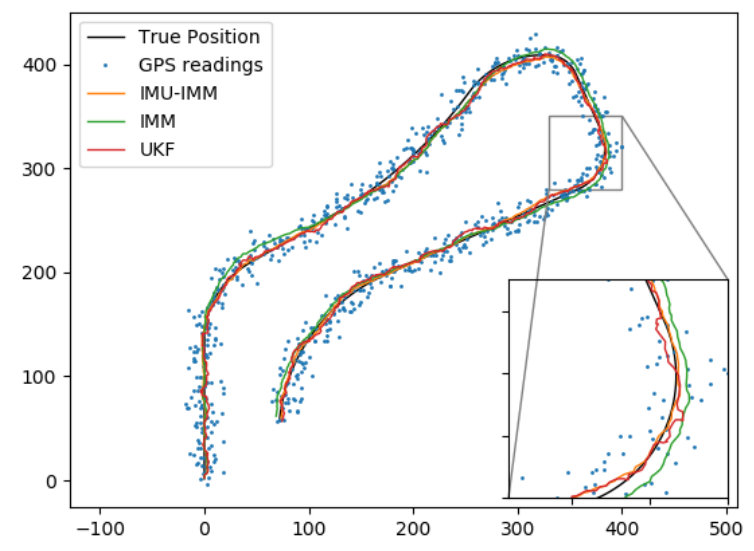

Fig. 4. Scenario 2: Position results for IMU-IMM, IMM and UKF.
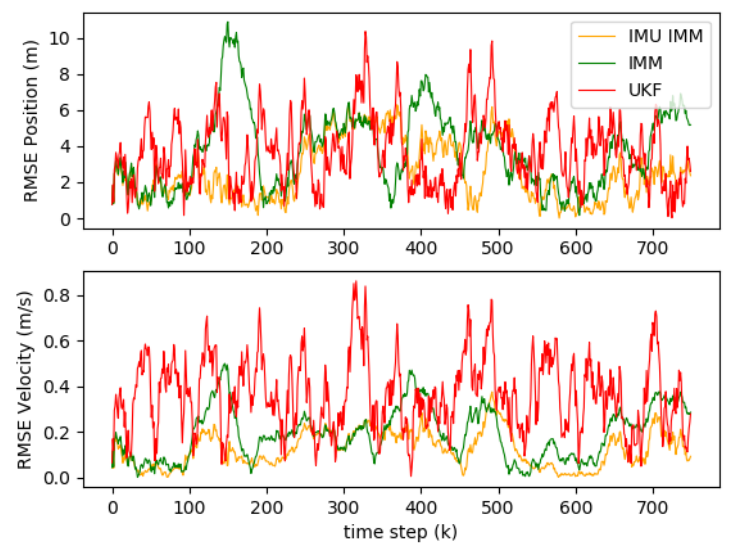

Fig. 5. Scenario 2: Position \& Velocity RMSE for IMU-IMM, IMU and UKF.
Quantitatively speaking, RMSE found in IMU-IMM is about $30 \%$ less than both UKF and IMM. For velocity, proposed algorithm is $65 \%$ and $35 \%$ better than UKF and IMM, respectively. Again, IMU-IMM differs negligibly to IMM in smoothness by just around $2 \%$ while performing exceptionally than UKF at 55\% improvement for position and $77 \%$ for velocity.

\section{Scenario 3}

Commands in Scenario 3 attempts to replicate common autonomous trials with short return missions such as environmental surveys. The route consists of a series of curved journeys intermediated by a couple of sharper right turns. The ordered and repeated movements allow the analysis of algorithm's consistency in adaptations.

As before, figures here preserve the same formatting. Fig. 6 visualises the nearly enclosed return path taken by the USV. IMM (green line) localisation starts eminently close to black line until it approaches the first turn and struggles to keep up, offsetting the stable trajectory away from the true state. UKF (red line) produces unsteady estimations around black line as expected, accumulating RMSE. Combination of the two positive attributes are displayed by the IMU-IMM (orange line); evident in the enlarged inset by performing smoother than UKF but as accurate. IMU-IMM's credibility in sustaining low error over the alternatives are strengthen in Fig. 7. Furthermore, in this scenario, it is just around $20 \%$ off in RMSCE from IMM.

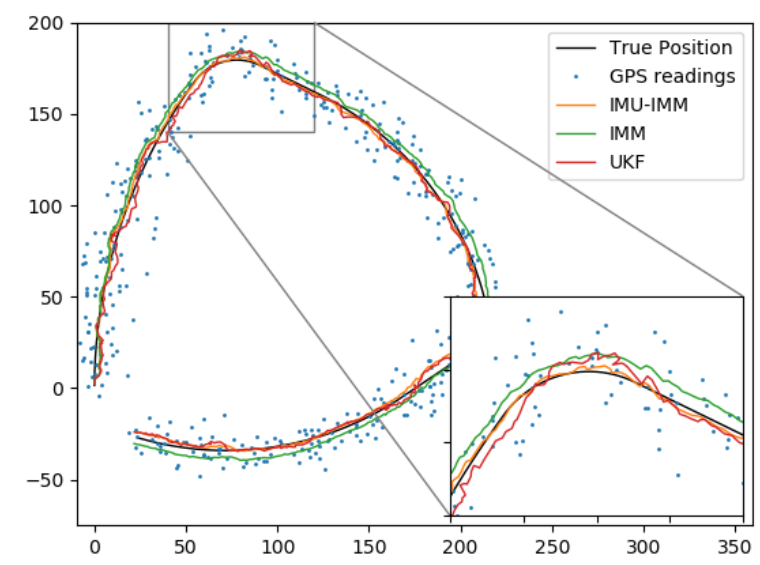

Fig. 6. Scenario 3: Position results for IMU-IMM, IMM and UKF.
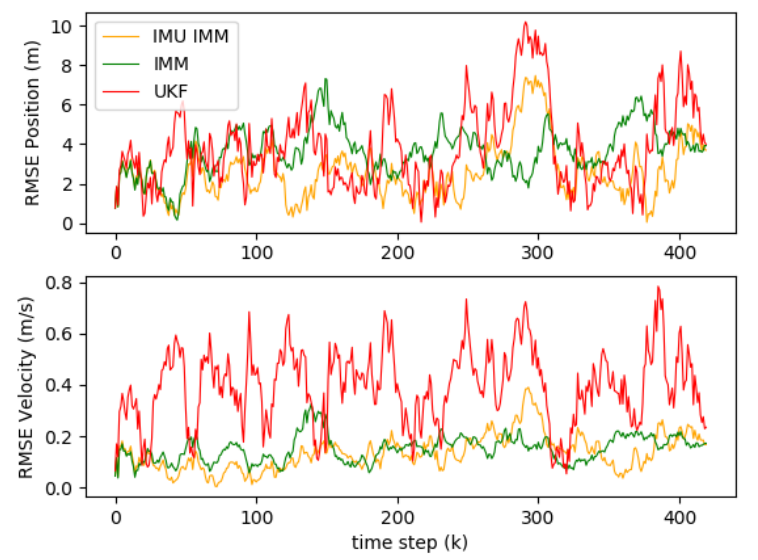

Fig. 7. Scenario 3: Position \& Velocity RMSE for IMU-IMM, IMU and UKF. 


\section{CONCLUSION}

This paper proposes the use of IMU-IMM for USV localisation using GPS/IMU and compass in practical setting. Contemporary single model filtering techniques lack reliability for USVs as its dependency on one model yield noisy results, implying that USVs are operating in an unpractical manner. IMM first replaces previous method. Then, minor readjustment to the IMM was proposed. With only small alterations, the IMU-IMM is able to exploit IMU's gyroscope that senses relevant mode change responsively. Adjunctive mode likeliness derived from the IMU is merged with IMM's mode probability, raising its confidence in assigning suitable mode. Theorised algorithm was examined in simulations based on USVs' pragmatic operations in comparison to UKF and IMM. IMM was proven to have desirable smoothness in its estimations than UKF but is poor in quickly responding to mode shifts. Through simulations, IMU-IMM was seen to produce states closer in overall to ground truth than IMM, while still possessing IMM's realistic localisation stability. Error evaluations taking account different scenarios indicate approximately $25 \%$ increase in accuracy to UKF and only a maximum of $10 \%$ difference in smoothness to IMM. Future work may be continued by adding adaptive measures to the possibility of poor a priori knowledge. Preferably, the adaptive feature should avoid more parametric definitions. Moreover, this research work would also benefit from having empirical validation.

\section{REFERENCES}

[1] Z. Liu, Y. Zhang, X. Yu and C. Yuan, "Unmanned surface vehicles: An overview of developments and challenges," Annual Reviews in Control, pp. 71-93, 2016.

[2] R. Sutton, S. Sharma and T. Xu, "Adaptive navigation systems for an unmanned surface vehicle," Journal of Marine Engineering \& Technology, vol. 10, no. 3, pp. 3-20, 2011.

[3] C. Hide, T. Moore and M. Smith, "Adaptive Kalman Filtering for Low-cost INS/GPS," The Journal of Navigation, vol. 56, p. 143-152, 2003.

[4] J. H. Ryu, G. Gankhuyag and K. T. Chong, "Navigation System Heading and Position Accuracy Improvement through GPS and INS Data Fusion," Journal of Sensors, 2016.

[5] W. Liu, Y. Liu, R. Song and R. Bucknall, "The Design of an Embedded Multi-Sensor Data Fusion System for Unmanned Surface Vehicle Navigation Based on Real Time Operating System," in 2018 OCEANS - MTS/IEEE Kobe Techno-Oceans (OTO), Kobe, Japan, 2018.

[6] D. Simon, Optimal State Estimation, John Wiley \& Sons, 2006.

[7] C. Hu, W. Chen, Y. Chen and D. Liu, "Adaptive Kalman Filtering for Vehicle Navigation," Journal of Global Positioning Systems, vol. 2, no. 1 , pp. 42-47, 2003.

[8] S. J. Julier and J. Uhlmann, "A New Extension of the Kalman Filter to Nonlinear Systems," Proc. SPIE 3068, Signal Processing, Sensor Fusion, and Target Recognition, 1997.

[9] E. Wan and R. V. D. Merwe, "The unscented Kalman filter for nonlinear estimation," in Proceedings of the IEEE 2000 Adaptive Systems for Signal Processing, Communications, and Control Symposium, Alberta, Canada, 2000.

[10] J. Shen, Y. Liu, S. Wang and Z. Sun, "Evaluation of Unscented Kalman Filter and Extended Kalman Filter for Radar Tracking Data Filtering," in European Modelling Symposium, Pisa, 2014.
[11] A. Almagbile, J. Wang and W. Ding, "Evaluating the Performances of Adaptive Kalman Filter Methods in GPS/INS Integration," Journal of Global Positioning Systems, vol. 9, no. 1, pp. 33-40, 2010.

[12] R. Mehra, "Approaches to adaptive filtering," IEEE Trans. Autom. Control, vol. 17, no. 5, pp. 693-698, 1972.

[13] J. Wang, "Stochastic Modeling for Real-Time Kinematic GPS/GLONASS Positioning," Navigation, vol. 46, no. 4, pp. 297-305, 1999.

[14] R. Fitzgerald, "Divergence of the Kalman filter," IEEE Transactions on Automatic Control, vol. 16, no. 6, pp. 736 - 747, 1971.

[15] Y. Meng, S. Gao, Y. Zhong, G. Hu and A. Subic, "Covariance matching based adaptive unscented Kalman filter for direct filtering in INS/GNSS integration," Acta Astronautica, vol. 120, pp. 171-181, 2016.

[16] B. Zheng, P. Fu, B. Li and X. Yuan, "A Robust Adaptive Unscented Kalman Filter for Nonlinear Estimation with Uncertain Noise Covariance," Sensors, vol. 18, no. 3, 2018.

[17] W. Liu, Y. Liu and R. Bucknall, "A Robust Localization Method for Unmanned Surface Vehicle (USV) Navigation Using Fuzzy Adaptive Kalman Filtering," IEEE Access, vol. 7, pp. 46071-46083, 2019.

[18] I. Hashlamon, "A New Adaptive Extended Kalman Filter for a Class of Nonlinear Systems," Journal of Applied and Computational Mechanics, vol. 6, no. 1, pp. 1-12, 2019.

[19] A. Assa and K. N. Plataniotis, "Adaptive Kalman Filtering by Covariance Sampling," IEEE Signal Processing Letters, vol. 24, no. 9, pp. 1288 - 1292, 2017.

[20] Y. Huang, Y. Zhang, Z. Wu, N. Li and J. Chambers, "A Novel Adaptive Kalman Filter With Inaccurate Process and Measurement Noise Covariance Matrices," IEEE Transactions on Automatic Control, vol. 63, no. 2, pp. 594-601, 2018.

[21] A. H. Mohamed and K. P. Schwarz, "Adaptive Kalman Filtering for INS/GPS," Journal of Geodesy, vol. 73, p. 193-203, 1999.

[22] J. Sasiadek, Q. Wang and M. Zeremba, "Fuzzy adaptive Kalman filtering for INS/GPS data fusion," in Proceedings of the 2000 IEEE International Symposium on Intelligent Control, Rio Patras, 2000.

[23] H. A. P. Blom and Y. Bar-Shalom, "The interacting multiple model algorithm for systems with Markovian switching coefficients," IEEE Transactions on Automatic Control, vol. 33, no. 8, pp. 780-783, 1998.

[24] Y. Liu, R. Song and R. Bucknall, "Intelligent Tracking of Moving Ships in Constrained Maritime Environments Using AIS," Cybernetics and Systems, 2019.

[25] X. R. Li and Y. Bar-Shalom, "Multiple-model estimation with variable structure," IEEE Transactions on Automatic Control, vol. 41, no. 4, pp. 478-493, 1996.

[26] J. B. B. Gomes, "An Overview on Target Tracking Using Multiple Model," Citeseer, 2008.

[27] X. R. Li and V. P. Jilkov, "A survey of maneuvering target trackingPart V: Multiple-model methods," IEEE Transactions on Aerospace and Electronic Systems, vol. 41, no. 4, pp. 1255 - 1321, 2005.

[28] Y. Bar-Shalom, X. R. Li and T. Kirubarajan, Estimation with Applications to Tracking and Navigation: Theory Algorithms and Software, New York: Wiley, 2001.

[29] E. Mazor, A. Averbuch, Y. Bar-Shalom and J. Dayan, "Interacting multiple model methods in target tracking: A survey," IEEE Transactions on Aerospace and Electronic Systems, vol. 34, no. 1, pp. 103-123, 1998.

[30] H. Qu, L. Pang and S. Li, "A novel interacting multiple model algorithm," Signal Process, vol. 89, no. 11, pp. 2171-2177, 2009.

[31] Y. Bar-Shalom, K.Chang and H. Blom, "Tracking of splitting targets in clutter using an interacting multiple model joint probabilistic data association filter," in Proc. of the 30th IEEE Conference on Decision and Control, Brighton, UK, 1991. 